\title{
Mobile Prisoner's Dilemma game played on diverse habitats
}

Citation for published version (APA):

Celebi, R., \& Kiliç, H. (2011). Mobile Prisoner's Dilemma game played on diverse habitats. In Evolutionary Computation (CEC), 2011 IEEE Congress on (pp. 2600-2604) https://doi.org/10.1109/CEC.2011.5949942

Document status and date:

Published: 01/01/2011

DOI:

10.1109/CEC.2011.5949942

Document Version:

Publisher's PDF, also known as Version of record

Document license:

Taverne

Please check the document version of this publication:

- A submitted manuscript is the version of the article upon submission and before peer-review. There can be important differences between the submitted version and the official published version of record.

People interested in the research are advised to contact the author for the final version of the publication, or visit the DOI to the publisher's website.

- The final author version and the galley proof are versions of the publication after peer review.

- The final published version features the final layout of the paper including the volume, issue and page numbers.

Link to publication

\footnotetext{
General rights rights.

- You may freely distribute the URL identifying the publication in the public portal. please follow below link for the End User Agreement:

www.umlib.nl/taverne-license

Take down policy

If you believe that this document breaches copyright please contact us at:

repository@maastrichtuniversity.nl

providing details and we will investigate your claim.
}

Copyright and moral rights for the publications made accessible in the public portal are retained by the authors and/or other copyright owners and it is a condition of accessing publications that users recognise and abide by the legal requirements associated with these

- Users may download and print one copy of any publication from the public portal for the purpose of private study or research.

- You may not further distribute the material or use it for any profit-making activity or commercial gain

If the publication is distributed under the terms of Article $25 \mathrm{fa}$ of the Dutch Copyright Act, indicated by the "Taverne" license above, 


\section{Mobile Prisoner's Dilemma Game Played On Diverse Habitats}

\author{
Remzi Çelebi \\ Space Technologies Research Institute \\ TUBITAK-SPACE, METU Campus \\ 06531, Ankara, Turkey \\ Email: remzi.celebi@uzay.tubitak.gov.tr
}

\author{
Hürevren Kılıç \\ Department of Computer Engineering \\ Atılım University \\ 06836, Incek, Ankara, Turkey \\ Email: hurevren@atilim.edu.tr
}

\begin{abstract}
Promotion of cooperative behavior in Prisoner's Dilemma (PD) game while players that are allowed to move between different gaming environments (i.e. habitats) is investigated. The stochastic mobile model under study is realized over connected habitats that are situated on a two dimensional grid environment. The players appearing in the same habitat are allowed to interact with their immediate neighbors. Mobility of a player is defined as movement from its habitat to another based on both obtained payoff and randomly assigned habitat diversity values. By the end of extensive experimentation, it is concluded that player mobility is an effective factor that contributes to promotion of cooperation in spatial evolutionary games. Also, even for higher values of temptation of PD game, habitat diversity supports and triggers a collective resistance for the emergence and promotion of cooperative system behavior.
\end{abstract}

Keywords : Evolutionary game theory, mobile prisoners dilemma, promotion of cooperation, habitat diversity.

\section{INTRODUCTION}

Cooperation is a common behavior observed in various systems like ecological, social, economic and biological systems. Individuals tend to cooperate with each other, even there is a cost of cooperation for them. Emergence of cooperation and promotion of altruistic behavior under the temptation of selfish defective behavior is a fundamental issue in such systems. The Prisoner's Dilemma is a well known metaphor to model such encounters among players in evolutionary game theory [1]. In PD game, there are two types of competing players; cooperators (Cs) that are inclined to cooperate and defectors (Ds) that do not prefer to cooperate but to exploit the cooperators.

Nowak and May [2] have first studied the impact of spatial structure and neighborhood interactions on cooperative cluster formation of individuals playing PD game. Following this work, investigation of relation between topological aspects of the population and promotion of cooperation due to spatial organization of situated players becomes the rationale behind the studies on spatial versions of the PD. The spatial PD versions include regular lattice, small-world networks and random graphs [3]-[4]. Scale-free networks [5] have been reported to have potential to promote cooperative behavior in prisoner's dilemma and snowdrift games [7],[8]. Scalefree topologies provide realistic representations to describe connectivity among communities. The preferential attachment mechanism is used to build scale-free networks. It is realized by setting local rules between individuals [9].

In this paper, we report the results of experimentation about evolution of cooperation among mobile players of PD game. The objective of the research is to examine the impact of player mobility on promotion of cooperation realized on diversified and connected habitat environments. Habitat diversity refers to different environmental conditions for alternative spatial locations. The connected and diverse habitat concept adds realism to the PD since the environment can be thought as a form of a player that affects the obtained welfare of the others. In our case, Mobile Prisoner's Dilemma (MPD) game setup realizes player migration due to obtained cumulative payoff and habitat diversity values. MPD provides more realistic and useful setup to investigate the dynamics of cooperative behavior.

The idea of introducing diversity to PD game is not a new one. In [10], the diversity among individual players' social status in society is represented by some scaling factor. Similarly in [11], they investigate how diversity in the way individuals assess their adverse social partners affects the evolution of cooperation. In MPD game a spatial/locational diversity rather than player attributed diversity is considered. Also, habitat scaling factors affect the game payoffs based on their figured out values. In [12], the potential effect of mobility on system equilibrium has been experimented in the game of chicken played on cellular spaces. In their model, competition for space among players rather than promotion of cooperation was the main concern behind the designed mechanism. In MPD game setup, a player in one habitat can move to another one depending on their obtained payoff and diversity values of neighboring habitats.

Scaling factors are drawn randomly from exponential distribution and the factor values remain fixed throughout each run. Positive (and negative) scaling factors increase (and decrease) the magnitude of payoff values while preserving the nature of PD game. The generated habitat values are uniformly distributed over a 2D grid. In other words, there is no spatial correlation between generated assigned habitat diversity values. Player mobility is formulated to answer the basic question: Who will Go to Where (WGW)?

The paper is organized as follows: In Section II, the MPD 


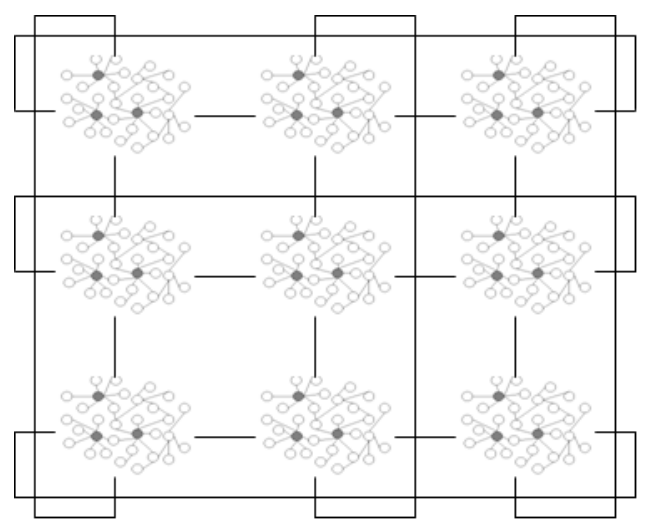

Fig. 1. An example playground for Mobile Prisoner's Dilemma (MPD) model: An L x L periodic boundary-conditioned 2-D lattice of scale-free habitat network with $\mathrm{L}=3$

game and properties of scaling factors that determine habitat diversity on the playground are defined; in Section III, the experimental setup and results are given and discussed. This section also includes some general comments about numerical implementations of PD based simulations. Section IV is the conclusions.

\section{MODEL DESCRIPTION}

An evolutionary two-strategy prisoner's dilemma game with players located initially on vertices of scale-free habitats is considered (see Figure 1). Each scale-free habitat is located on vertices of a two-dimensional square lattice of size $\mathrm{L} \times \mathrm{L}$ with periodic boundary conditions. The playground is a network of habitats on which individuals are allowed to interact with their neighbors residing in the same habitat. Players move from the habitats depending on their performance and the diversity of the habitat they reside.

At any simulation cycle, individuals adopt either defection $D$ or cooperation $C$ as strategy. The system evolves by applying interaction, imitation and migration phases one after another. During interaction phase, players encounter with each of their neighbors. In the PD game, each player adopts either a defecting strategy $\mathrm{D}$ or a cooperating strategy $\mathrm{C}$ and payoff values are computed accordingly. Mutual cooperation among players is rewarded $R$, but mutual defection results in punishment $P$. When a player prefers to cooperate but the other one does not, the player takes suckers payoff $S$ and the tempted defective player gets whatever the assigned temptation value $T$ is.

In the setup, we take original $T=b>1, R=1$ and $S$ $=P=0$ values and rescaled them according to habitat social status (diversity) while preserving the original games payoff ranking $(T>R>P>S)$. Rescaling on the game played at habitat $i$ is done according to similar formulation developed in [10]. In the original formula, the scaling acts as a mapping of the original payoffs to individual fitness values defining social diversity among individuals.

Rescaled payoffs have the form $U_{i j}^{r}=U_{i j}\left(1+h_{i}\right)$ where $j$ is a player residing at habitat $i, h_{i}$ is the scaling factor assigned to each habitat location at the beginning of simulations. $h_{i}$ is drawn randomly from exponential distributed habitat diversity defined by equation

$$
h=a(-\log \chi-1)
$$

where $\chi$ is a uniformly distributed random variable taking values from unit interval such that the average of $h$ values is zero. Note that, $\int_{0}^{1} h(\chi) d \chi=0$ so that average of $h$ over all the habitat locations is zero. Parameter $a$ in Equation (1) defines the amplitude of scaling factors. The degree of dispersion is defined by this parameter. When $a=0$, the original PD payoff values are obtained. On the other hand, when $a=1,\left(1+h_{i}\right) \geq 0$ preserved for all $i$ and possible PD payoff ranking changes are disabled.

During imitation phase, players update their strategy, synchronously. The whole population updates the strategies simultaneously in discrete time steps, giving rise to a discrete time dynamics on macroscopic level. A player $j$ located on habitat $i$ chooses one of its neighbors say $k$ as its cultural parent and copies its strategy $s_{i k}$ with a probability

$$
p_{s_{i j} \rightarrow s_{i k}}=\frac{1}{1+e^{\left(U_{i j}-U_{i k}\right) / K_{1}}}
$$

where $U_{i j}$ is the obtained payoff at one game cycle by player $j, U_{i k}$ is the payoff of randomly chosen neighbor $k$ in the same habitat $i$ and $K_{1}$ is the extent of noise [4].

The stochastic effects on the evolution of cooperation in MPD game are extended while introducing diversity of habitats and player mobility as two new model extensions. The habitat diversity is provided by introducing scaling factors that modify the game payoffs to scaled values. Note that the game played is still preserved to be the prisoner's dilemma while the game places are supposed to affect the obtained payoff values.

During migration phase, players migrate from one habitat to another based on their success and diversity of the habitat they reside. We can answer the two questions i) Who should leave from the current habitat? ii) Where can be the new location of the mover in terms of both its new habitat and its new neighbors in that new scale-free habitat location?

Related to the first question: In order to establish a relation between obtained payoff and player mobility we need to propose a mechanism. In our case, for a rescaled payoff $U_{i j}^{r}$, the probability of player $j$ at habitat $i$ to leave its location is computed by

$$
p_{i j}=1-\frac{U_{i j}^{r}}{M\left(U_{i j}^{r}\right)}
$$

where $M\left(U_{i j}^{r}\right)$ is the maximum payoff that player $j$ can retain in one game cycle played from its neighbors. Clearly, $M\left(U_{i j}^{r}\right)$ shows variation depending on the players current strategy. For cooperators it is calculated by

$$
M\left(U_{i j}^{r}\right)=k R\left(1+h_{i}\right)
$$


and for the defectors, by

$$
M\left(U_{i j}^{r}\right)=k T\left(1+h_{i}\right)
$$

where $k$ is the number of current neighbors of $j$. Players with higher payoff values are expected to continue their survival on their habitats. As a special case, when only one player resides in a habitat, it does not move to its neighbor habitats but stays. Note that in an habitat when all the players are cooperators, each player gets the maximum obtainable payoff and this results in $p_{i j}=0$ and no move from the habitat. Related to the second question: Decided movers in habitat $i$ can change their habitats by considering their four-neighboring habitats. The scaling factor $h_{i}$ of the habitat of the mover is compared with that of a randomly chosen neighbor habitats scaling factor $h_{j}$ and the probability to change its location from $i$ to $j$ is calculated by

$$
p_{i \rightarrow j}=\frac{1}{1+e^{\left(h_{i}-h_{j}\right) / K_{2}}}
$$

where $K_{2}$ is the extent of noise defining the uncertainty about player move process.

Formation and update of a scale-free habitat is critical for our simulations. Barabasi-Albert scale-free network generation algorithm [6] suggests two main ingredients of selforganization of a network: (i) growth followed by (ii) preferential attachment. A newcomer is connected to current topology based on preferential attachment algorithm. By this way, scalefree property of habitat is preserved against possible changes due to player mobility. In simulations, the number of nodes that newcomer attached is assumed to be 4 . If a habitat holds less than 4 players, then the newcomer is attached to all.

We know that scale-free networks are robust to random failures like node collapse (or removal) but fragile to intentional attacks [13][14]. For the removal of node $i$, we propose the following simple procedure inspired from preferential attachment. Let $\Omega_{i}$ be the set of neighbors of node $i$ and $k_{i}$ show degree of node $i$ :

- Step 1: Remove node $i$ together with its connected edges.

- Step 2: Decide on node $j$ taken from $\Omega_{i}$ based on probability:

$$
\Pi_{j}=\frac{k_{j}}{\sum_{l \in \Omega_{i}} k_{l}}
$$

- Step 3: Connect node $j$ to all other nodes in $\Omega_{i}$ (except itself), if exists.

The above procedure prevents potential graph disconnections caused by node removals. However, an evaluation about its possible scale-free preserving property still requires some mathematical elaboration.

\section{Simulation Setup, Results And Discussions}

This section is divided into three subsections. The first subsection defines the designed simulation setup. In the second one, simulation results are reported and discussed. The last subsection contains general comments about both numerical implementations of PD based simulations and result interpretations.

\section{A. The Setup}

IVC software [15] id used to generate initial scale-free habitats. Habitats are generated by initial 4 core nodes extended by 16 attached nodes each with 4 neighbors. $K_{1}$ and $K_{2}$ parameters, in Equations (2) and (4) define uncertainties in strategy change and movement from habitat processes, respectively. They are both assumed to be 0.05 . Scaling factors are drawn randomly from a given distribution to determine the diversity of habitat places. The habitat grid size was 20times 20 . Two different sizes $N=200$ and 500 are tried as the initial population for each habitat. For all runs initial cooperator density values (FC) are set with 0.5 probabilities at individual habitat level. By the end of 1000 iterations of all runs, the FC results are observed to converge. For each different habitat diversity and temptation values, 20 independent simulations are executed. 11 different amplitude values ranging from 0.0 to 1.0 in 0.1 step size and 11 different temptation values ranging from 1.0 to 2.0 in 0.1 step size are considered. Therefore, total number of independent runs is: $11 \cdot 11 \cdot 20 \cdot 2=4840$ for each of two initial habitat population sizes. Runs for immobile mode (as the base model) are also taken. Total number of runs for the immobile mode is: $11 \cdot 20$. Each simulation run is performed on a single computational node of a cluster. The system is implemented in Java programming language.

\section{B. Results and Discussions}

Figure 2 depicts the values of cooperator densities (FC) on the strategy state space which is calculated by taking the average over 20 independent simulations for each amplitude and temptation values. We observed that the existence of both habitat diversity (i.e. $a>0.0$ ) and mobility promotes higher cooperation rate than the immobile case for temptation values $b>=1.7$. For $a=0.0$ and $b>=1.5$, there are no cooperators left in the society by the end of 1000 iteration cycles of all 20 independent runs. Nonexisting habitat diversity implies a flat grid landscape and allows random movement. However, grid landscape with diverse habitat values provides a kind of probabilistic guidance for cooperators to reach potential locations populated with cooperators. In immobile case, the population size in a habitat is crucial factor for survival of cooperation and it requires enough number of individuals reside in the same habitat. For any setup either immobile, nondiverse or diverse habitat, when temptation values are as low as $b<1.5$, the cooperators dominate the society. The reason for that is simply the scale-free nature of the habitats. See [7] for compatible results.

Figure 3 gives an idea about general behavior and convergence of an individual run for various $a$ and $b$ values with initial population sizes 200 and 500. The FC values show convergence by the end of 1000 iteration cycles. A common characteristic of all 20 independent runs is a decrease in FC values in first 20-25 iterations of both immobile, diverse and nondiverse habitats during which mobility of players are also observed to be high. Such behavior can be explained by the initial dominance of defectors over cooperators in every habitat, which is suppressed relatively by later formation of 

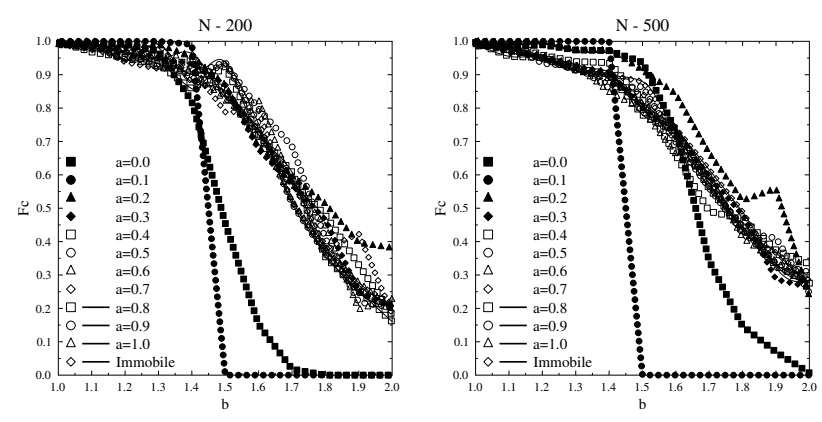

Fig. 2. Cooperator density (FC) values on the temptation-amplitude (b-a) parameter space where $\mathrm{N}=200,500$.

cooperation. As it is expected, in all runs, cooperator densities decrease down for higher temptation values.

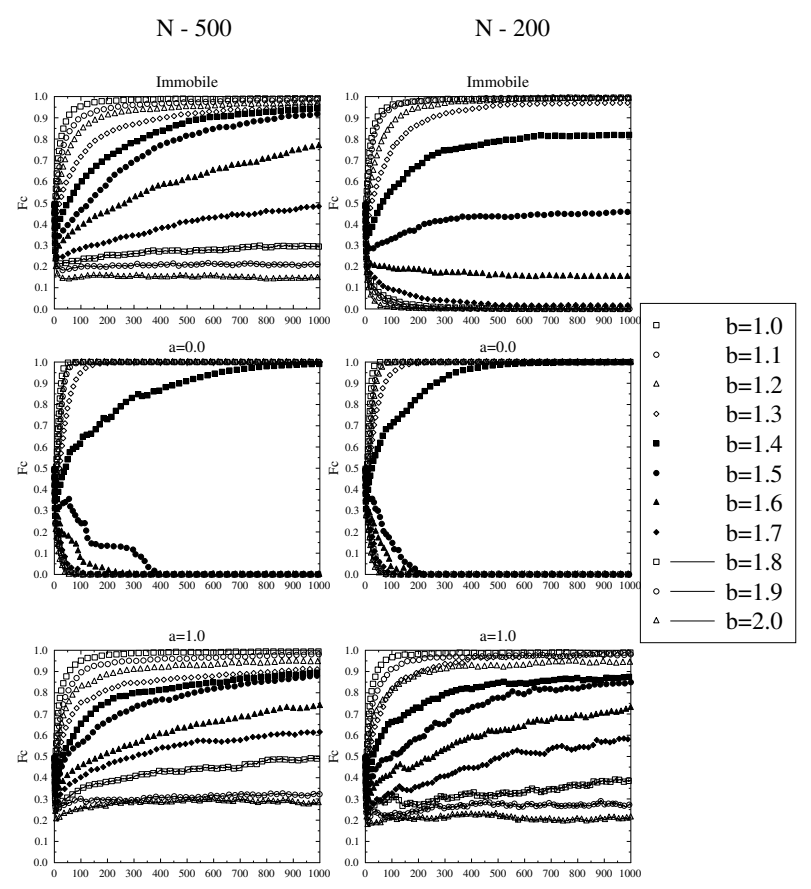

Iteration Number

Fig. 3. Cooperator density (FC) changes throughout iteration cycles for various amplitude (a) and temptation (b) settings. Simulations were carried out for $\mathrm{N}=500$ (left column), $\mathrm{N}=200$ (right column) values.

Since players are mobile, it would be interesting to examine their potential spatial distribution by the end of 1000 iteration cycles. Figure 4 and 5 show linear color scaled values indicating the number of cooperators and defectors for the runs of Figure 3. Note that, white dots in figure represent at most one cooperator or defector player residing in a habitat. There is no considerable difference between the results in Figure 4 and Figure 5. This means initial population size of habitats has no important effect on the final spatial distribution of players. The highest habitat diversity $a=1.0$ together with mobility promote the cooperation even for high temptation values. The same effect can not be observed for nondiverse $a=0.0$ and immobile cases especially when $b=2.0$. In immobile setup the promotion results become more sensitive to initial population sizes. When $b=1.0$, in any case cooperation is the dominant strategy and the observed blank habitats in two mobile cases are simply due to the provided mobility.

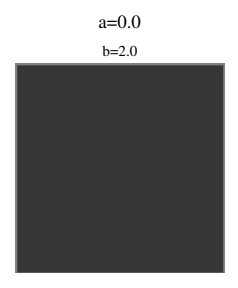

$\mathrm{b}=1.5$
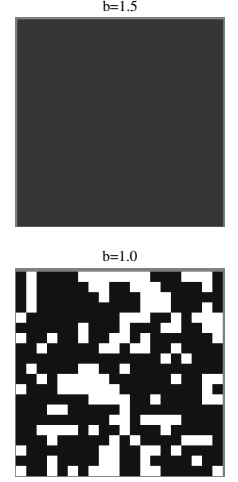
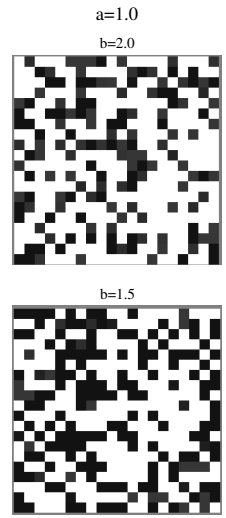

$\mathrm{b}=1.0$

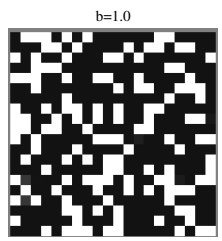

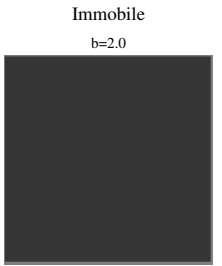

$b=1.5$

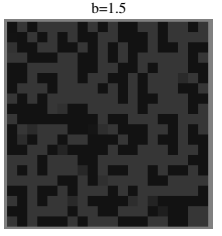

$b=1.0$

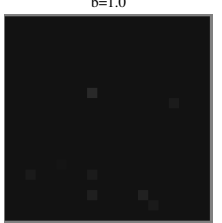

Fig. 4. Spatial distribution of Defective (Gray) and Cooperative (Black) players by the end of 1000 iteration cycles for the run given in Figure 3 for selected immobile mode, diversity $a=(0.0,1.0)$ and temptation $b=$ $(1.0,1.5,2.0)$ values where $\mathrm{N}=200$.

\section{General Comments}

In [16], it has been pointed out that the outcome of socalled games on grids depends somewhat on their numerical implementation. As PD model becomes more complex and the number of model initialization parameters and model stochastic components are high, the simulations may not reveal hidden system behavior. Another factor that can limit the observability of potential system behavior is the quality of random number generator used in simulations. Cycles in random number generation process can limit the investigation of whole encounter space and this may prevent the emergence of potential system behavior. Furthermore, synchronous gaming based on iteration cycles seems an important assumption that may effect the conclusions drawn about the system behavior.

PD game is not the only mechanism to explain actually found altruism/cooperation among individuals and societies. For example, a simple alternative description for cooperation realized on graphs and social networks has been pointed in [17]. Kin selection, reciprocal altruism and more sophisticated processes exemplify different perspectives to the problem. Beside from this, the results obtained in this study (or in general any other PD based simulations) should not be over generalized especially when making judgments about human societies whose behavior is known (or at least felt) to be 


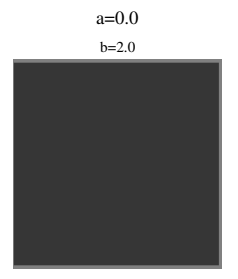

$b=1.5$

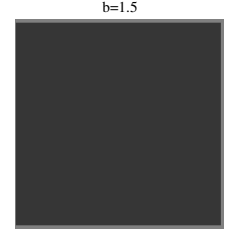

$\mathrm{b}=1.0$

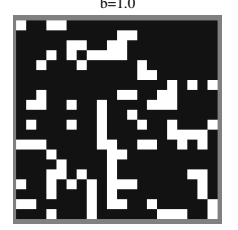

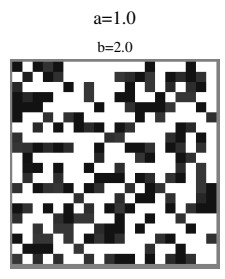

$b=1.5$

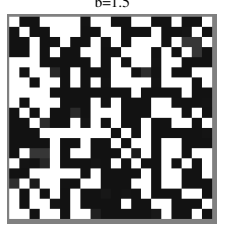

$\mathrm{b}=1.0$

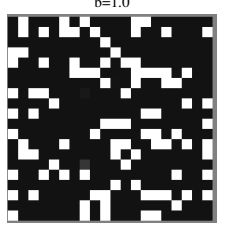

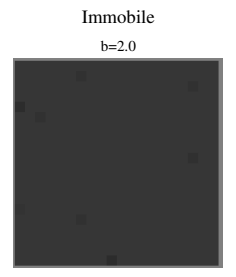

$b=1.5$

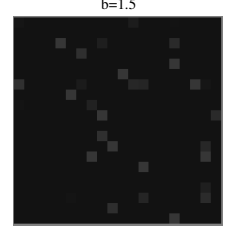

$b=1.0$

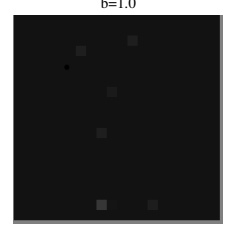

Fig. 5. Spatial distribution of Defective (Gray) and Cooperative (Black) players by the end of 1000 iteration cycles for the run given in Figure 3 for selected immobile mode, diversity $a=(0.0,1.0)$ and temptation $b=$ $(1.0,1.5,2.0)$ values where $\mathrm{N}=500$.

complex enough. Specifically, playing D or C may not be a reflection of selfish or altruistic preference of the player but it may just be a consequence of his/her adoption of honesty as the third strategy that drives his/her conscious decision. Therefore, careless interpretations about macroscopic system behavior based on $\mathrm{D}$ and $\mathrm{C}$ label statistics can be misleading when trying to explain complex social interactions.

\section{CONCLUSIONS}

A new cooperation promotion model MPD game in diverse habitats is introduced. Specifically, we have investigated how the introduction of habitat diversity and particularly its amplitude affect the evolution of cooperation amongst players moving on a spatial 2-D grid network of scale-free habitats. Mobile player model provided systematic and stochastic formulation of dynamically changing neighborhood description. It is observed that an existence of habitat diversity together with mobility provide promotion of cooperation even for high temptation values. Alternatively, in immobile mode, promotion of cooperation can only be achieved when the scale-free network habitats are populated enough. Finally, we point out that especially for human social systems, one must be careful about associating meanings to the conclusions drawn by the end of even so extensive simulations since the identifiability of human intentions from the actions is still questionable.

\section{REFERENCES}

[1] R. M. Axelrod, The evolution of cooperation. New York: Basic Books, 1984.

[2] M. A. Nowak and R. M. May, "Evolutionary games and spatial chaos," Nature, vol. 359, no. 6398, pp. 826-829, October 1992.
[3] M. N. and A. K., "Spatial prisoner's dilemma optimally played in smallworld networks," Physics Letters A, vol. 313, pp. 55-61(7), 23 June 2003.

[4] G. Szabó and C. Töke, "Evolutionary prisoner's dilemma game on a square lattice," Phys. Rev. E, vol. 58, no. 1, pp. 69-73, 1998.

[5] A. L. Barabasi and R. Albert, "Emergence of scaling in random networks," Science, vol. 286, pp. 509-512, 1999.

[6] Reka, Albert and Barabási "Statistical mechanics of complex networks,", Rev. Mod. Phys. vol. 74, pp.47-97, 2002.

[7] F. C. Santos and J. M. Pacheco, "Scale-free networks provide a unifying framework for the emergence of cooperation," Phys. Rev. Lett., vol. 95, no. 9, p. 098104, Aug 2005.

[8] F. C. Santos, J. M. Pacheco, and T. Lenaerts, "Evolutionary dynamics of social dilemmas in structured heterogeneous populations," Proceedings of the National Academy of Sciences of the United States of America, vol. 103, no. 9, pp. 3490-3494, February 2006.

[9] S. N. Dorogovtsev and J. F. F. Mendes, Evolution of Networks: From Biological Nets to the Internet and $W W W$. Oxford University Press, March 2003.

[10] M. Perc and A. Szolnoki, "Social diversity and promotion of cooperation in the spatial prisoner's dilemma game," Physical Review E, vol. 77, p. 011904, 2008.

[11] S. V. Segbroeck, F. C. Santos, T. Lenaerts, and J. M. Pacheco, "Emergence of cooperation in adaptive social networks with behavioral diversity," European Conference on Artificial Life 2009 (ECAL 2009) Budapest, Hungary. to be published in Lecture Notes In Computer Science (LNCS) Series in, 2010.

[12] P. Andrade, A. Monteiro, G. Camara, and S.Sandri, "Games on cellular spaces: How mobility affects equilibrium," Journall of Artificial Socities and Socila Simulation, vol. 12, no. 1 5, p. January, 2009.

[13] D. S. Callaway, M. E. J. Newman, S. H. Strogatz, and D. J. Watts, "Network robustness and fragility: Percolation on random graphs," Physical Review Letters, vol. 85, p. 5468, 2000.

[14] R. Cohen, K. Erez, D. ben Avraham, and S. Havlin, "Resilience of the internet to random breakdowns," Physical Review Letters, vol. 85, p. 4626, 2000.

[15] "Information Visualization Cyber Infrastructure" http://iv.slis.indiana.edu.

[16] M. R. Frean, “The prisoner's dilemma without synchrony," Biological Sciences, vol. 257, pp. 75-79, 1994.

[17] H. Ohtsuki, C. Hauert, E. Lieberman, and M. A. Nowak, "A simple rule for the evolution of cooperation on graphs and social networks," Nature, vol. 441, no. 7092, pp. 502-505, 2006. 